\title{
Existence and Attractivity for Fractional Evolution Equations
}

\author{
Yong Zhou $\mathbb{D}^{1,2}$, Jia Wei He, ${ }^{1}$ Bashir Ahmad, ${ }^{2}$ and Ahmed Alsaedi ${ }^{2}$ \\ ${ }^{1}$ Faculty of Mathematics and Computational Science, Xiangtan University, Hunan 411105, China \\ ${ }^{2}$ Nonlinear Analysis and Applied Mathematics (NAAM) Research Group, Faculty of Science, King Abdulaziz University, \\ Jeddah 21589, Saudi Arabia
}

Correspondence should be addressed to Yong Zhou; yzhou@xtu.edu.cn

Received 31 August 2017; Accepted 6 December 2017; Published 10 January 2018

Academic Editor: Francisco R. Villatoro

Copyright (C) 2018 Yong Zhou et al. This is an open access article distributed under the Creative Commons Attribution License, which permits unrestricted use, distribution, and reproduction in any medium, provided the original work is properly cited.

We study the existence and attractivity of solutions for fractional evolution equations with Riemann-Liouville fractional derivative. We establish sufficient conditions for the global attractivity of mild solutions for the Cauchy problems in the case that semigroup is compact.

\section{Introduction}

Fractional differential equations have gained considerable importance due to their application in various sciences, such as physics, mechanics, chemistry, and engineering. In recent years, there has been a significant development on ordinary and partial differential equations involving fractional derivatives; see the monographs of Podlubny [1], Kilbas et al. [2], Diethelm [3], Tarasov [4], and Zhou [5, 6] and a series of papers [7-28] and the references cited therein.

Recently, Zhou [7], Chen et al. [19], Losada et al. [20], and Banas and O'Regan [21] investigated the attractivity of solutions for fractional ordinary differential equations and integral equations. On the other hand, the existence theory of solutions for time fractional evolution equations has been investigated intensively by many authors; for example, see Kim et al. [16], Bazhlekova [22], Wang et al. [23], Zacher [24], and Zhou et al. [25]. However, to the best of our knowledge, there are no results on the attractivity of solutions for fractional evolution equations in the literature.

Consider fractional evolution equation with RiemannLiouville derivative:

$$
\begin{aligned}
& { }^{L} D_{0+}^{\alpha} x(t)=A x(t)+f(t, x(t)), \quad t \in[0, \infty), \\
& I_{0+}^{1-\alpha} x(0)=x_{0},
\end{aligned}
$$

where ${ }^{L} D_{0+}^{\alpha}$ is Riemann-Liouville fractional derivative of order $0<\alpha<1, I_{0+}^{1-\alpha}$ is Riemann-Liouville fractional integral of order $1-\alpha, A$ is the infinitesimal generator of a $C_{0}$ semigroup of bounded linear operators $\{Q(t)\}_{t \geq 0}$ in Banach space $X, f:[0, \infty) \times X \rightarrow X$ is a given function satisfying some assumptions, and $x_{0}$ is an element of the Banach space $X$.

In this paper, we initiate the question of the attractivity of solutions for Cauchy problem (1). We establish sufficient conditions for the global attractivity for mild solutions of (1) in the case that semigroup is compact. These results essentially reveal the characteristics of solutions for fractional evolution equations with Riemann-Liouville derivative. More precisely, integer order evolution equations do not have such attractivity.

\section{Preliminaries}

In this section, we firstly recall some concepts on fractional integrals and derivatives and then give some lemmas which are useful in next sections.

Let $\alpha \in(0,1)$ and $u \in L^{1}([0, \infty), X)$. The RiemannLiouville fractional integral is defined by

$$
I_{0+}^{\alpha} u(t)=g_{\alpha}(t) * u(t)=\int_{0}^{t} g_{\alpha}(t-s) u(s) d s, \quad t>0,
$$

where $*$ denotes the convolution,

$$
g_{\alpha}(t)=\frac{t^{\alpha-1}}{\Gamma(\alpha)},
$$


and in case $\alpha=0$, we set $g_{0}(t)=\delta(t)$, the Dirac measure is concentrated at the origin. For $u \in C([0, \infty), X)$, the Riemann-Liouville fractional derivative is defined by

$$
{ }^{L} D_{0+}^{\alpha} u(t)=\frac{d}{d t}\left(g_{1-\alpha}(t) * u(t)\right) .
$$

The Wright function $M_{\alpha}(\theta)$ is defined by

$$
M_{\alpha}(\theta)=\sum_{n=1}^{\infty} \frac{(-\theta)^{n-1}}{(n-1) ! \Gamma(1-\alpha n)} .
$$

It is known that $M_{\alpha}(\theta)$ satisfies the following equality:

$$
\int_{0}^{\infty} \theta^{\delta} M_{\alpha}(\theta) d \theta=\frac{\Gamma(1+\delta)}{\Gamma(1+\alpha \delta)}, \quad \text { for } \delta \geq 0 .
$$
(1).

We give the following definition of the mild solution of

Definition 1 (see [5]). By the mild solution of the Cauchy problem (1), we mean that the function $x \in C([0, \infty), X)$ satisfies

$$
\begin{aligned}
x(t)= & t^{\alpha-1} P_{\alpha}(t) x_{0} \\
& +\int_{0}^{t}(t-s)^{\alpha-1} P_{\alpha}(t-s) f(s, x(s)) d s, \quad t>0,
\end{aligned}
$$

where

$$
P_{\alpha}(t)=\int_{0}^{\infty} \alpha \theta M_{\alpha}(\theta) Q\left(t^{\alpha} \theta\right) d \theta .
$$

Definition 2. The mild solution $x(t)$ of the Cauchy problem (1) is attractive if $x(t)$ tends to zero as $t \rightarrow \infty$.

Suppose that $A$ is the infinitesimal generator of a $C_{0^{-}}$ semigroup $\{Q(t)\}_{t \geq 0}$ of uniformly bounded linear operators on Banach space $X$. This means that there exists $M \geq 1$ such that

$$
M=\sup _{t \in[0, \infty)}\|Q(t)\|_{B(X)}<\infty,
$$

where $B(X)$ be the space of all bounded linear operators from $X$ to $X$ with the norm $\|Q\|_{B(X)}=\sup \{|Q(x)|:|x|=1\}$, where $Q \in B(X)$ and $x \in X$.

Proposition 3 (see [5]). For any fixed $t>0, P_{\alpha}(t)$ is linear and bounded operator, that is, for any $x \in X$,

$$
\left|P_{\alpha}(t) x\right| \leq \frac{M}{\Gamma(\alpha)}|x| .
$$

Proposition 4 (see [5]). $\left\{P_{\alpha}(t)\right\}_{t>0}$ is strongly continuous, which means that, for $\forall x \in X$ and $t^{\prime \prime}>t^{\prime}>0$, we have

$$
\left|P_{\alpha}\left(t^{\prime \prime}\right) x-P_{\alpha}\left(t^{\prime}\right) x\right| \longrightarrow 0 \quad \text { as } t^{\prime \prime} \longrightarrow t^{\prime} .
$$

Proposition 5 (see [5]). Assume that $\{Q(t)\}_{t>0}$ is compact operator. Then $\left\{P_{\alpha}(t)\right\}_{t>0}$ is also compact operator.
Let $X$ be a real Banach space, $J=[0, \infty)$ :

$$
E=\left\{y \in C(J, X): \lim _{t \rightarrow \infty} \frac{|y(t)|}{1+t}=0\right\},
$$

with the norm $\|y\|=\sup _{t \in[0, \infty)}|y(t)| /(1+t)$. It is easy to see that $(E,\|\cdot\|)$ is a Banach space.

We need also the following generalization of AscoliArzela theorem, which one can find in [29].

Lemma 6. The set $\mathscr{H} \subset E$ is relatively compact if and only if the following conditions hold:

(i) For any $b>0$, the function in $U=\{y: y(t)=x(t) /(1+$ $t), x \in \mathscr{H}\}$ is equicontinuous on $[0, b]$.

(ii) For any $t \in[0, \infty), U(t)=\mathscr{H}(t) /(1+t)$ is relatively compact in $X$.

(iii) $\lim _{t \rightarrow \infty}|x(t)| /(1+t)=0$ uniformly for $x \in \mathscr{H}$.

Theorem 7 (Schauder fixed point theorem). Let $S$ be a nonempty, closed, and convex subset of the Banach space $X$ and let $F: S \rightarrow$ S be completely continuous; then $F$ has a fixed point in $S$.

Lemma 8 (see [26]). If $\alpha, \mu, \omega>0$, then

$$
\int_{0}^{t}(t-s)^{\alpha-1} s^{\mu-1} \mathrm{e}^{-\omega s} d s \leq C t^{\alpha-1}, \quad t>0
$$

where $C=\max \left\{1,2^{1-\alpha}\right\} \Gamma(\mu)(1+\mu(\mu+1) / \alpha) \omega^{-\mu}>0$.

\section{Some Lemmas}

In this paper, we always suppose that the operator $A$ generates a compact $C_{0}$-semigroup $\{Q(t)\}_{t>0}$ on $X$; that is, the operator $Q(t)$ is compact for $t>0$.

Let

$$
\begin{array}{r}
C_{\alpha}(J, X)=\left\{x \in C((0, \infty), X): \lim _{t \rightarrow 0+} t^{1-\alpha} x(t)\right. \\
\text { exists and is finite, } \left.\lim _{t \rightarrow \infty} \frac{t^{1-\alpha}|x(t)|}{1+t}=0\right\},
\end{array}
$$

with the norm

$$
\|x\|_{\alpha}=\sup _{t \in[0, \infty)} \frac{t^{1-\alpha}|x(t)|}{1+t} .
$$

Then $\left(C_{\alpha}(J, X),\|\cdot\|_{\alpha}\right)$ is a Banach space by the similar proof of [27, Lemma 3.2].

For any $x \in C_{\alpha}(J, X)$, consider the operator $F$ defined by

$$
(F x)(t)=\left(F_{1} x\right)(t)+\left(F_{2} x\right)(t),
$$

where

$$
\begin{aligned}
& \left(F_{1} x\right)(t)=t^{\alpha-1} P_{\alpha}(t) x_{0}, \quad \text { for } t \in(0, \infty), \\
& \left(F_{2} x\right)(t)=\int_{0}^{t}(t-s)^{\alpha-1} P_{\alpha}(t-s) f(s, x(s)) d s,
\end{aligned}
$$


It is easy to see $\lim _{t \rightarrow 0^{+}} t^{1-\alpha}(F x)(t)=x_{0} / \Gamma(\alpha)$. It is clear that $x$ is a mild solution of $(1)$ in $C_{\alpha}(J, X)$ if and only if there exists a fixed point $x^{*} \in C_{\alpha}(J, X)$, such that $x^{*}=F x^{*}$ holds.

For any $y \in E$, let

$$
x(t)=t^{\alpha-1} y(t), \quad \text { for } t \in(0, \infty) .
$$

Then, $x \in C_{\alpha}(J, X)$. Define an operator $\mathscr{F}$ as follows:

$$
(\mathscr{F} y)(t)=\left(\mathscr{F}_{1} y\right)(t)+\left(\mathscr{F}_{2} y\right)(t)
$$

where

$$
\begin{aligned}
& \left(\mathscr{F}_{1} y\right)(t)= \begin{cases}t^{1-\alpha}\left(F_{1} x\right)(t), & \text { for } t \in(0, \infty), \\
\frac{x_{0}}{\Gamma(\alpha)}, & \text { for } t=0,\end{cases} \\
& \left(\mathscr{F}_{2} y\right)(t)= \begin{cases}t^{1-\alpha}\left(F_{2} x\right)(t), & \text { for } t \in(0, \infty), \\
0, & \text { for } t=0 .\end{cases}
\end{aligned}
$$

In this section, we always suppose that the following condition holds:

(H1) There exist $K \geq 0$ and $\beta_{1} \in(\alpha, 1)$, such that $|f(t, x(t))| \leq K t^{-\beta_{1}}$, for any $x \in C((0, \infty), X)$.

Let $\gamma_{1} \in\left(0, \beta_{1}\right)$. Then there exists $T_{1}>0$ such that

$$
\frac{M\left|x_{0}\right|}{\Gamma(\alpha)} T_{1}^{\gamma_{1}-1}+\frac{M K \Gamma\left(1-\beta_{1}\right)}{\Gamma\left(1+\alpha-\beta_{1}\right)} T_{1}^{\gamma_{1}-\beta_{1}} \leq 1 .
$$

Let

$$
S_{1}=\left\{y: y \in E,\left|\frac{t^{\gamma_{1}} y(t)}{1+t}\right| \leq 1 \text {, for } t \geq T_{1}\right\} \text {. }
$$

Before obtaining our main results, we firstly prove some lemmas as follows.

Lemma 9. Assume that (H1) holds. Then, $\{U y: U y(t)=$ $\left.(\mathscr{F} y)(t) /(1+t), y \in S_{1}\right\}$ is equicontinuous and $\lim _{t \rightarrow \infty}|U y(t)|=0$ uniformly for $y \in S_{1}$.

Proof. It is clear that $S_{1}$ is a nonempty, closed, and convex subset of $E$.

Claim I. $\left\{U y: U y(t)=\left(\mathscr{F}_{1} y\right)(t) /(1+t), y \in S_{1}\right\}$ is equicontinuous.

For $t_{1}=0, t_{2} \in(0, \infty)$, we have

$$
\begin{aligned}
& \left|\frac{\left(\mathscr{F}_{1} y\right)\left(t_{2}\right)}{1+t_{2}}-\left(\mathscr{F}_{1} y\right)(0)\right| \\
& \quad \leq\left|\frac{1}{1+t_{2}} P_{\alpha}\left(t_{2}\right) x_{0}-\frac{x_{0}}{\Gamma(\alpha)}\right| \\
& \quad \leq\left|\frac{1}{1+t_{2}} P_{\alpha}\left(t_{2}\right)-\frac{1}{\Gamma(\alpha)}\right|\left|x_{0}\right| \longrightarrow 0, \text { as } t_{2} \longrightarrow 0 .
\end{aligned}
$$

For any $t_{1}, t_{2} \in(0, \infty)$ and $t_{1}<t_{2}$, we have

$$
\begin{aligned}
& \left|\frac{\left(\mathscr{F}_{1} y\right)\left(t_{2}\right)}{1+t_{2}}-\frac{\left(\mathscr{F}_{1} y\right)\left(t_{1}\right)}{1+t_{1}}\right| \\
& \leq\left|\frac{P_{\alpha}\left(t_{2}\right) x_{0}}{1+t_{2}}-\frac{P_{\alpha}\left(t_{1}\right) x_{0}}{1+t_{1}}\right| \\
& \leq\left|\frac{P_{\alpha}\left(t_{2}\right) x_{0}}{1+t_{2}}-\frac{P_{\alpha}\left(t_{2}\right) x_{0}}{1+t_{1}}\right| \\
& \quad+\left|\frac{P_{\alpha}\left(t_{2}\right) x_{0}}{1+t_{1}}-\frac{P_{\alpha}\left(t_{1}\right) x_{0}}{1+t_{1}}\right| \\
& \leq\left|P_{\alpha}\left(t_{2}\right)\right|\left|x_{0}\right| \frac{\left|t_{2}-t_{1}\right|}{\left(1+t_{2}\right)\left(1+t_{1}\right)} \\
& \quad+\left|P_{\alpha}\left(t_{2}\right)-P_{\alpha}\left(t_{1}\right)\right|\left|x_{0}\right| \frac{1}{1+t_{1}} \longrightarrow 0,
\end{aligned}
$$

Hence, $\left\{U y: U y(t)=\left(\mathscr{F}_{1} y\right)(t) /(1+t), y \in S_{1}\right\}$ is equicontinuous.

Claim II. $\left\{U y: U y(t)=\left(\mathscr{F}_{2} y\right)(t) /(1+t), y \in S_{1}\right\}$ is equicontinuous. For any $y \in S_{1}$, let $x(t)=t^{\alpha-1} y(t), t \in$ $(0, \infty)$. Then $x \in \widetilde{S}_{1}$, where

$$
\begin{aligned}
& \widetilde{S}_{1} \\
& \quad=\left\{x \in C_{\alpha}(J, X):\left|\frac{t^{\gamma_{1}} t^{1-\alpha} x(t)}{1+t}\right| \leq 1, \text { for } t \geq T_{1}\right\},
\end{aligned}
$$

which is nonempty, closed, and convex.

Since $\alpha<\beta_{1}<1$ and $\varepsilon>0$ is given, then there exists $T^{\prime}>0$ enough large, such that

$$
\frac{M K \Gamma\left(1-\beta_{1}\right)}{\Gamma\left(1+\alpha-\beta_{1}\right)} \frac{T^{\prime 1-\beta_{1}}}{1+T^{\prime}}<\frac{\varepsilon}{2} .
$$

For $t_{1}, t_{2}>T^{\prime}$, in virtue of (H1) and (26), we get

$$
\begin{aligned}
& \left|\frac{\left(\mathscr{F}_{2} y\right)\left(t_{2}\right)}{1+t_{2}}-\frac{\left(\mathscr{F}_{2} y\right)\left(t_{1}\right)}{1+t_{1}}\right| \\
& \leq\left|\frac{t_{2}^{1-\alpha}}{1+t_{2}} \int_{0}^{t_{2}}\left(t_{2}-s\right)^{\alpha-1} P_{\alpha}\left(t_{2}-s\right) f(s, x(s)) d s\right| \\
& \quad+\left|\frac{t_{1}^{1-\alpha}}{1+t_{1}} \int_{0}^{t_{1}}\left(t_{1}-s\right)^{\alpha-1} P_{\alpha}\left(t_{1}-s\right) f(s, x(s)) d s\right| \\
& \leq \frac{M K}{\Gamma(\alpha)} \frac{t_{2}^{1-\alpha}}{1+t_{2}} \int_{0}^{t_{2}}\left(t_{2}-s\right)^{\alpha-1} s^{-\beta_{1}} d s \\
& \quad+\frac{M K}{\Gamma(\alpha)} \frac{t_{1}^{1-\alpha}}{1+t_{1}} \int_{0}^{t_{1}}\left(t_{1}-s\right)^{\alpha-1} s^{-\beta_{1}} d s \\
& \leq \frac{2 M K \Gamma\left(1-\beta_{1}\right)}{\Gamma\left(1+\alpha-\beta_{1}\right)} \frac{T^{\prime 1-\beta_{1}}}{1+T^{\prime}}<\varepsilon, \quad \text { as } t_{2} \longrightarrow t_{1} .
\end{aligned}
$$


For $t_{1}=0,0<t_{2} \leq T^{\prime}$, we have

$$
\begin{aligned}
& \left|\frac{\left(\mathscr{F}_{2} y\right)\left(t_{2}\right)}{1+t_{2}}-\left(\mathscr{F}_{2} y\right)(0)\right| \\
& \quad=\left|\frac{t_{2}^{1-\alpha}}{1+t_{2}} \int_{0}^{t_{2}}\left(t_{2}-s\right)^{\alpha-1} P_{\alpha}\left(t_{2}-s\right) f(s, x(s)) d s\right| \\
& \quad \leq \frac{M K}{\Gamma(\alpha)} \frac{t_{2}^{1-\alpha}}{1+t_{2}} \int_{0}^{t_{2}}\left(t_{2}-s\right)^{\alpha-1} s^{-\beta_{1}} d s \\
& \quad \leq \frac{M K \Gamma\left(1-\beta_{1}\right)}{\Gamma\left(1+\alpha-\beta_{1}\right)} \frac{t_{2}^{1-\beta_{1}}}{1+t_{2}} \rightarrow 0 \text { as } t_{2} \longrightarrow 0 .
\end{aligned}
$$

For $0<t_{1}<t_{2} \leq T^{\prime}$, we have

$$
\begin{aligned}
& \left|\frac{\left(\mathscr{F}_{2} y\right)\left(t_{2}\right)}{1+t_{2}}-\frac{\left(\mathscr{F}_{2} y\right)\left(t_{1}\right)}{1+t_{1}}\right| \leq \mid \frac{t_{1}^{1-\alpha}}{1+t_{1}} \int_{t_{1}}^{t_{2}}\left(t_{2}-s\right)^{\alpha-1} \\
& \cdot P_{\alpha}\left(t_{2}-s\right) f(s, x(s)) d s|+| \frac{t_{1}^{1-\alpha}}{1+t_{1}} \\
& \quad \cdot \int_{0}^{t_{1}}\left(\left(t_{2}-s\right)^{\alpha-1}-\left(t_{1}-s\right)^{\alpha-1}\right) P_{\alpha}\left(t_{2}-s\right) \\
& \cdot f(s, x(s)) d s|+| \frac{t_{1}^{1-\alpha}}{1+t_{1}} \int_{0}^{t_{1}}\left(t_{1}-s\right)^{\alpha-1} \\
& \cdot\left(P_{\alpha}\left(t_{2}-s\right)-P_{\alpha}\left(t_{1}-s\right)\right) f(s, x(s)) d s \mid \\
& \quad+\left|\frac{t_{2}^{1-\alpha}}{1+t_{2}}-\frac{t_{1}^{1-\alpha}}{1+t_{1}}\right| \mid \int_{0}^{t_{2}}\left(t_{2}-s\right)^{\alpha-1} P_{\alpha}\left(t_{2}-s\right) \\
& \quad \cdot f(s, x(s)) d s \mid \leq I_{1}+I_{2}+I_{3}+I_{4},
\end{aligned}
$$

where

$$
\begin{aligned}
I_{1}= & \frac{M K}{\Gamma(\alpha)} \frac{t_{1}^{1-\alpha}}{1+t_{1}} \mid \int_{0}^{t_{2}}\left(t_{2}-s\right)^{\alpha-1} s^{-\beta_{1}} d s \\
& -\int_{0}^{t_{1}}\left(t_{1}-s\right)^{\alpha-1} s^{-\beta_{1}} d s \mid, \\
I_{2}= & \frac{2 M K}{\Gamma(\alpha)} \frac{t_{1}^{1-\alpha}}{1+t_{1}} \int_{0}^{t_{1}}\left(\left(t_{1}-s\right)^{\alpha-1}-\left(t_{2}-s\right)^{\alpha-1}\right) \\
& \cdot s^{-\beta_{1}} d s, \\
I_{3}= & \frac{t_{1}^{1-\alpha}}{1+t_{1}} \mid \int_{0}^{t_{1}}\left(t_{1}-s\right)^{\alpha-1}\left(P_{\alpha}\left(t_{2}-s\right)-P_{\alpha}\left(t_{1}-s\right)\right) \\
& \cdot f(s, x(s)) d s \mid, \\
I_{4}= & \left|\frac{t_{2}^{1-\alpha}}{1+t_{2}}-\frac{t_{1}^{1-\alpha}}{1+t_{1}}\right| \frac{M K \Gamma\left(1-\beta_{1}\right)}{\Gamma\left(1+\alpha-\beta_{1}\right)} t_{2}^{\alpha-\beta_{1}} .
\end{aligned}
$$

It can deduce that $I_{1} \rightarrow 0$ as $t_{2} \rightarrow t_{1}$ directly. Indeed,

$$
\begin{aligned}
I_{1} & =\frac{M K \Gamma\left(1-\beta_{1}\right)}{\Gamma\left(1+\alpha-\beta_{1}\right)} \frac{t_{1}^{1-\alpha}\left|t_{2}^{\alpha-\beta_{1}}-t_{1}^{\alpha-\beta_{1}}\right|}{1+t_{1}} \\
& \leq \frac{M K \Gamma\left(1-\beta_{1}\right)}{\Gamma\left(1+\alpha-\beta_{1}\right)} \frac{t_{1}^{1-\beta_{1}} t_{2}^{\alpha-\beta_{1}}\left(t_{2}-t_{1}\right)^{\beta_{1}-\alpha}}{1+t_{1}} \longrightarrow 0,
\end{aligned}
$$

$$
\text { as } t_{2} \longrightarrow t_{1} \text {. }
$$

Note that

$$
\left(\left(t_{1}-s\right)^{\alpha-1}-\left(t_{2}-s\right)^{\alpha-1}\right) s^{-\beta_{1}} \leq\left(t_{1}-s\right)^{\alpha-1} s^{-\beta_{1}},
$$$$
\text { for } s \in\left[0, t_{1}\right] \text {, }
$$

and the map $s \rightarrow\left(t_{1}-s\right)^{\alpha-1} s^{-\beta_{1}}$ is integrable for $s \in\left[0, t_{1}\right]$ and $t_{1} \in\left[0, T^{\prime}\right]$, then by Lebesgue dominated convergence theorem, we have

$$
\begin{aligned}
\int_{0}^{t_{1}}\left(\left(t_{1}-s\right)^{\alpha-1}-\left(t_{2}-s\right)^{\alpha-1}\right) s^{-\beta_{1}} d s \longrightarrow & 0, \\
& \text { as } t_{2} \longrightarrow t_{1},
\end{aligned}
$$

which implies that $I_{2} \rightarrow 0$ as $t_{2} \rightarrow t_{1}$.

For given $\varepsilon>0$ small enough, from the condition (H1), we get

$$
\begin{aligned}
I_{3} & \leq \frac{t_{1}^{1-\alpha}}{1+t_{1}} \int_{0}^{t_{1}-\varepsilon}\left(t_{1}-s\right)^{\alpha-1} \\
& \cdot\left|\left(P_{\alpha}\left(t_{2}-s\right)-P_{\alpha}\left(t_{1}-s\right)\right) f(s, x(s))\right| d s \\
& +\frac{t_{1}^{1-\alpha}}{1+t_{1}} \int_{t_{1}-\varepsilon}^{t_{1}}\left(t_{1}-s\right)^{\alpha-1} \\
& \cdot\left|\left(P_{\alpha}\left(t_{2}-s\right)-P_{\alpha}\left(t_{1}-s\right)\right) f(s, x(s))\right| d s \\
& \leq \frac{t_{1}^{1-\alpha}}{1+t_{1}} \int_{0}^{t_{1}}\left(t_{1}-s\right)^{\alpha-1}|f(s, x(s))| d s \\
& \cdot \sup _{s \in\left[0, t_{1}-\varepsilon\right]}\left|P_{\alpha}\left(t_{2}-s\right)-P_{\alpha}\left(t_{1}-s\right)\right|+\frac{t_{1}^{1-\alpha}}{1+t_{1}} \frac{2 M}{\Gamma(\alpha)} \\
& \cdot \int_{t_{1}-\varepsilon}^{t_{1}}\left(t_{1}-s\right)^{\alpha-1}|f(s, x(s))| d s \leq I_{31}+I_{32}+I_{33}
\end{aligned}
$$

where

$$
\begin{array}{r}
I_{31}=\frac{t_{1}^{1-\alpha}}{1+t_{1}} \int_{0}^{t_{1}}\left(t_{1}-s\right)^{\alpha-1}|f(s, x(s))| d s \\
\cdot \sup _{s \in\left[0, t_{1}-\varepsilon\right]}\left\|P_{\alpha}\left(t_{2}-s\right)-P_{\alpha}\left(t_{1}-s\right)\right\|_{B(X)},
\end{array}
$$




$$
\begin{aligned}
I_{32} & =\frac{t_{1}^{1-\alpha}}{1+t_{1}} \frac{2 M K}{\Gamma(\alpha)} \\
& \cdot \int_{0}^{t_{1}-\varepsilon}\left(\left(t_{1}-\varepsilon-s\right)^{\alpha-1}-\left(t_{1}-s\right)^{\alpha-1}\right) s^{-\beta_{1}} d s, \\
I_{33} & =\frac{t_{1}^{1-\alpha}}{1+t_{1}} \frac{2 M K}{\Gamma(\alpha)} \mid \int_{0}^{t_{1}}\left(t_{1}-s\right)^{\alpha-1} s^{-\beta_{1}} d s \\
& -\int_{0}^{t_{1}-\varepsilon}\left(t_{1}-\varepsilon-s\right)^{\alpha-1} s^{-\beta_{1}} d s \mid .
\end{aligned}
$$

By Proposition 4 , we have that $I_{31} \rightarrow 0$ as $t_{2} \rightarrow t_{1}$. Similar to the proof that $I_{1}, I_{2}$ tend to zero, we get $I_{32} \rightarrow 0$ and $I_{33} \rightarrow 0$ as $\varepsilon \rightarrow 0$. Thus, we get that $I_{3}$ tend to zero independently of $y \in S_{1}$ as $t_{2} \rightarrow t_{1}, \varepsilon \rightarrow 0$.

Similar to (24), it is easy to proof that $I_{4} \rightarrow 0$ as $t_{2} \rightarrow t_{1}$.

For $0 \leq t_{1}<T^{\prime}<t_{2}$, note that if $t_{2} \rightarrow t_{1}$, then $t_{2} \rightarrow T^{\prime}$ and $t_{1} \rightarrow T^{\prime}$, from the above argument, we obtain

$$
\begin{aligned}
& \left|\frac{\left(\mathscr{F}_{2} y\right)\left(t_{2}\right)}{1+t_{2}}-\frac{\left(\mathscr{F}_{2} y\right)\left(t_{1}\right)}{1+t_{1}}\right| \\
& \leq\left|\frac{\left(\mathscr{F}_{2} y\right)\left(t_{2}\right)}{1+t_{2}}-\frac{\left(\mathscr{F}_{2} y\right)\left(T^{\prime}\right)}{1+T^{\prime}}\right| \\
& \quad+\left|\frac{\left(\mathscr{F}_{2} y\right)\left(T^{\prime}\right)}{1+T^{\prime}}-\frac{\left(\mathscr{F}_{2} y\right)\left(t_{1}\right)}{1+t_{1}}\right| \longrightarrow 0,
\end{aligned}
$$

Therefore, it is clear that

$$
\left|\frac{\left(\mathscr{F}_{2} y\right)\left(t_{2}\right)}{1+t_{2}}-\frac{\left(\mathscr{F}_{2} y\right)\left(t_{1}\right)}{1+t_{1}}\right| \longrightarrow 0, \quad \text { as } t_{2} \longrightarrow t_{1},
$$

independently of $y \in S_{1}$. Thus, $\left\{U y: U y(t)=\left(\mathscr{F}_{2} y\right)(t) /(1+\right.$ $\left.t), y \in S_{1}\right\}$ is equicontinuous..

Claim III. $\lim _{t \rightarrow \infty}|U y(t)|=0$ uniformly for $y \in S_{1}$. For any $y \in S_{1}$, by (H1) and Proposition 3 , we have

$$
\begin{aligned}
& |(\mathscr{F} y)(t)| \\
& \leq\left|P_{\alpha}(t) x_{0}\right| \\
& \quad+\left|t^{1-\alpha} \int_{0}^{t}(t-s)^{\alpha-1} P_{\alpha}(t-s) f(s, x(s)) d s\right| \\
& \quad \leq \frac{M\left|x_{0}\right|}{\Gamma(\alpha)}+\frac{M K}{\Gamma(\alpha)} \int_{0}^{t} t^{1-\alpha}(t-s)^{\alpha-1} s^{-\beta_{1}} d s \\
& \quad \leq \frac{M\left|x_{0}\right|}{\Gamma(\alpha)}+\frac{M K \Gamma\left(1-\beta_{1}\right)}{\Gamma\left(1+\alpha-\beta_{1}\right)} t^{1-\beta_{1}} .
\end{aligned}
$$

We multiply the above inequalities at both sides with the factor $1 /(1+t)$, then

$$
\frac{|(\mathscr{F} y)(t)|}{1+t} \leq \frac{M\left|x_{0}\right|}{\Gamma(\alpha)} \frac{1}{1+t}+\frac{M K \Gamma\left(1-\beta_{1}\right)}{\Gamma\left(1+\alpha-\beta_{1}\right)} \frac{t^{1-\beta_{1}}}{1+t}
$$

and, therefore,

$$
\begin{aligned}
\frac{|(\mathscr{F} y)(t)|}{1+t} & \leq \frac{M\left|x_{0}\right|}{\Gamma(\alpha)} \frac{1}{1+t}+\frac{M K \Gamma\left(1-\beta_{1}\right)}{\Gamma\left(1+\alpha-\beta_{1}\right)} \frac{t^{1-\beta_{1}}}{1+t} \\
& \longrightarrow 0, \text { as } t \longrightarrow \infty,
\end{aligned}
$$

which shows that $\lim _{t \rightarrow \infty}|U y(t)|=0$ uniformly for $y \in S_{1}$. The proof is completed.

Lemma 10. Assume that (H1) holds. Then $\mathscr{F}$ maps $S_{1}$ into itself and $\mathscr{F}$ is continuous.

Proof.

Claim I. F maps $S_{1}$ into $S_{1}$. For any $y \in S_{1}$, let $x(t)=t^{\alpha-1} y(t)$, $t \in(0, \infty)$. Then $x \in \widetilde{S}_{1}$. that

For $t \geq T_{1}$, together inequity (21) and $0<\gamma_{1}<\beta_{1}$ yield

$$
\begin{aligned}
& \frac{M\left|x_{0}\right|}{\Gamma(\alpha)} \frac{t^{\gamma_{1}}}{1+t}+\frac{M K \Gamma\left(1-\beta_{1}\right)}{\Gamma\left(1+\alpha-\beta_{1}\right)} \frac{t^{1-\beta_{1}+\gamma_{1}}}{1+t} \\
& \quad \leq \frac{M\left|x_{0}\right|}{\Gamma(\alpha)} t^{\gamma_{1}-1}+\frac{M K \Gamma\left(1-\beta_{1}\right)}{\Gamma\left(1+\alpha-\beta_{1}\right)} t^{\gamma_{1}-\beta_{1}} \\
& \quad \leq \frac{M\left|x_{0}\right|}{\Gamma(\alpha)} T_{1}^{\gamma_{1}-1}+\frac{M K \Gamma\left(1-\beta_{1}\right)}{\Gamma\left(1+\alpha-\beta_{1}\right)} T_{1}^{\gamma_{1}-\beta_{1}} \leq 1 .
\end{aligned}
$$

Then, for $t \geq T_{1}$, from (39), we have

$$
\begin{aligned}
\frac{\left|t^{\gamma_{1}}(\mathscr{F} y)(t)\right|}{1+t} \leq & \frac{M\left|x_{0}\right|}{\Gamma(\alpha)} \frac{t^{\gamma_{1}}}{1+t} \\
& +\frac{M K \Gamma\left(1-\beta_{1}\right)}{\Gamma\left(1+\alpha-\beta_{1}\right)} \frac{t^{1-\beta_{1}+\gamma_{1}}}{1+t} \leq 1,
\end{aligned}
$$

which deduce that $\mathscr{F} S_{1} \subset S_{1}$ for $t \geq T_{1}$.

Claim II. $\mathscr{F}$ is continuous. For any $y_{m}, y \in S_{1}, m=$ $1,2, \ldots$, with $\lim _{m \rightarrow \infty} y_{m}=y$, let $x(t)=t^{\alpha-1} y(t), x_{m}(t)=$ $t^{\alpha-1} y_{m}(t), t \in(0, \infty)$. Then $x_{m}, x \in \widetilde{S}_{1}$.

In addition, we have

$$
\begin{gathered}
\lim _{m \rightarrow \infty} y_{m}(t)=y(t), \\
\lim _{m \rightarrow \infty} t^{\alpha-1} y_{m}(t)=t^{\alpha-1} y(t),
\end{gathered}
$$

for $t \in(0, \infty)$.

By (H1) and the continuity of $f$ on any compact subsets uniformly, then we get

$$
\begin{aligned}
\lim _{m \rightarrow \infty} f\left(t, x_{m}(t)\right) & =\lim _{m \rightarrow \infty} f\left(t, t^{\alpha-1} y_{m}(t)\right) \\
& =f\left(t, t^{\alpha-1} y(t)\right)=f(t, x(t)) .
\end{aligned}
$$

Therefore, for a.e. $t \in[0, T],(t-s)^{\alpha-1} \mid f\left(s, x_{m}(s)\right)-$ $f(s, x(s)) \mid \leq 2 L(t-s)^{\alpha-1} s^{-\beta_{1}}$ via (H1). In addition, the function $s \rightarrow 2 L(t-s)^{\alpha-1} s^{-\beta_{1}}$ is integrable for $s \in[0, t]$ 
and $t \in[0, T]$. Hence, by Lebesgue dominated convergence theorem, we obtain

$$
\int_{0}^{t}(t-s)^{\alpha-1}\left|f\left(s, x_{m}(s)\right)-f(s, x(s))\right| d s \longrightarrow 0,
$$

as $m \longrightarrow \infty$.

Then, for $t \in[0, T]$,

$$
\begin{aligned}
& \left|\frac{\left(\mathscr{F} y_{m}\right)(t)}{1+t}-\frac{(\mathscr{F} y)(t)}{1+t}\right| \leq \frac{t^{1-\alpha}}{1+t} \int_{0}^{t}(t-s)^{\alpha-1} \\
& \cdot\left|P_{\alpha}(t-s)\left(f\left(s, x_{m}(s)\right)-f(s, x(s))\right)\right| d s \\
& \leq \frac{M}{\Gamma(\alpha)} \frac{t^{1-\alpha}}{1+t} \int_{0}^{t}(t-s)^{\alpha-1} \\
& \cdot\left|f\left(s, x_{m}(s)\right)-f(s, x(s))\right| d s,
\end{aligned}
$$

which yields that $\left\|\mathscr{F} y_{m}-\mathscr{F} y\right\| \rightarrow 0$ as $m \rightarrow \infty$.

On the other hand, let $\varepsilon>0$ be given, fixed enough large $T>0$ with replaced $T^{\prime}$ such that (26) holds. Then, for $t>T$, by virtue of (26) and (27), we have

$$
\begin{aligned}
& \left|\frac{\left(\mathscr{F} y_{m}\right)(t)}{1+t}-\frac{(\mathscr{F} y)(t)}{1+t}\right| \leq \frac{M}{\Gamma(\alpha)} \frac{t^{1-\alpha}}{1+t} \\
& \quad \cdot \int_{0}^{t}(t-s)^{\alpha-1}\left(\left|f\left(s, x_{m}(s)\right)\right|+|f(s, x(s))|\right) d s \\
& \quad \leq \frac{2 M K \Gamma\left(1-\beta_{1}\right)}{\Gamma\left(1+\alpha-\beta_{1}\right)} \frac{T^{1-\beta_{1}}}{1+T}<\varepsilon .
\end{aligned}
$$

Therefore, it is obvious that $\left\|\mathscr{F} y_{m}-\mathscr{F} y\right\| \rightarrow 0$ as $m \rightarrow$ $\infty$. Combined with the above statement, It can imply that $\mathscr{F} y_{m} \rightarrow \mathscr{F} y$ uniformly on $[0, \infty)$ as $m \rightarrow \infty$; that is, $\mathscr{F}$ is continuous.

\section{Main Results}

Theorem 11. Assume that $Q(t)(t>0)$ is compact, and the condition (H1) holds. Then the Cauchy problem (1) admits at least one attractive solution.

Proof. Obviously, $x$ is a mild solution of (1) in $\widetilde{S}_{1}$ if and only if $y$ is a fixed point of $y=\mathscr{F} y$ in $S_{1}$, where $x(t)=t^{\alpha-1} y(t)$. Thus, it is sufficient to show that $y=\mathscr{F} y$ has a fixed point in $S_{1}$. By Lemma 9, we know that $\{U y: U y(t)=(\mathscr{F} y)(t) /(1+t), y \in$ $\left.S_{1}\right\}$ is equicontinuous and $\lim _{t \rightarrow \infty}|U y(t)|=0$ uniformly for $y \in S_{1}$. It remains to verify that, for any $t \in[0, \infty), U y(t)$ is relatively compact in $X$ according to Lemma 6 . Obviously, $U y(0)$ is relatively compact in $X$. Let $t \in(0, \infty)$ be fixed. For every $\varepsilon \in(0, t)$ and $\delta>0$, define an operator $\mathscr{F}_{\varepsilon, \delta}$ on $S_{1}$ as follows:

$$
\begin{gathered}
U_{\varepsilon, \delta} y(t)=\frac{\left(\mathscr{F}_{\varepsilon, \delta} y\right)(t)}{1+t}=\frac{P_{\alpha}(t) x_{0}}{1+t}+\int_{0}^{t-\varepsilon} \int_{\delta}^{\infty} \alpha \theta \\
\cdot \frac{t^{1-\alpha}(t-s)^{\alpha-1}}{1+t} M_{\alpha}(\theta) Q\left((t-s)^{\alpha} \theta\right)
\end{gathered}
$$

$$
\begin{aligned}
& \cdot f(s, x(s)) d \theta d s=\frac{P_{\alpha}(t) x_{0}}{1+t}+Q\left(\varepsilon^{\alpha} \delta\right) \\
& \cdot \int_{0}^{t-\varepsilon} \int_{\delta}^{\infty} \alpha \theta \frac{t^{1-\alpha}(t-s)^{\alpha-1}}{1+t} M_{\alpha}(\theta) \\
& \cdot Q\left((t-s)^{\alpha} \theta-\varepsilon^{\alpha} \delta\right) f(s, x(s)) d \theta d s .
\end{aligned}
$$

Since $Q(t)$ is compact for $t>0$, by Proposition 5, we know that $P_{\alpha}(t)$ is compact. In addition, from the compactness of $Q\left(\varepsilon^{\alpha} \delta\right)$, we obtain that the set $\left\{U_{\varepsilon, \delta} y, y \in S_{1}\right\}$ is relatively compact in $X$ for any $\varepsilon \in(0, t)$ and for any $\delta>0$. For every $y \in S_{1}$, we have

$$
\begin{aligned}
& \left|U y(t)-U_{\varepsilon, \delta} y(t)\right| \leq \frac{t^{1-\alpha}}{1+t} \mid \int_{0}^{t-\varepsilon} \int_{0}^{\delta} \alpha \theta(t-s)^{\alpha-1} \\
& \cdot M_{\alpha}(\theta) Q\left((t-s)^{\alpha} \theta\right) f(s, x(s)) d \theta d s \mid \\
& +\frac{t^{1-\alpha}}{1+t} \mid \int_{t-\varepsilon}^{t} \int_{0}^{\infty} \alpha \theta(t-s)^{\alpha-1} M_{\alpha}(\theta) Q\left((t-s)^{\alpha} \theta\right) \\
& \cdot f(s, x(s)) d \theta d s \mid \leq \frac{\alpha M t^{1-\alpha}}{1+t} \int_{0}^{t-\varepsilon}(t-s)^{\alpha-1} \\
& \cdot|f(s, x(s))| d s \int_{0}^{\delta} \theta M_{\alpha}(\theta) d \theta+\frac{\alpha M t^{1-\alpha}}{1+t} \\
& \cdot \int_{t-\varepsilon}^{t}(t-s)^{\alpha-1}|f(s, x(s))| d s \int_{0}^{\infty} \theta M_{\alpha}(\theta) d \theta \\
& \leq \frac{\alpha M K t^{1-\beta_{1}}}{1+t} \frac{\Gamma(\alpha) \Gamma\left(1-\beta_{1}\right)}{\Gamma\left(1+\alpha-\beta_{1}\right)} \int_{0}^{\delta} \theta M_{\alpha}(\theta) d \theta \\
& +\frac{\alpha M K t^{1-\alpha}}{1+t} \int_{1-\varepsilon / t}^{1}(1-s)^{\alpha-1} s^{-\beta_{1}} d s \int_{0}^{\infty} \theta M_{\alpha}(\theta) d \theta \\
& \longrightarrow 0, \quad \text { as } \varepsilon \longrightarrow 0, \delta \longrightarrow 0 .
\end{aligned}
$$

Therefore, the set $U y(t)$ is closed to an arbitrary compact set. As a result, the set $U y(t)$ is also relatively compact set in $X$ for $t \in[0, \infty)$. By Lemma 6 , we know that $\mathscr{F} S_{1}$ is a relatively compact set. On the other hand, by Lemma 10 , we know that $\mathscr{F}$ maps $S_{1}$ into itself and $\mathscr{F}$ is continuous. Hence, $\mathscr{F}$ is a completely continuous operator. Therefore, according to Schauder fixed point theorem, there exists at least one fixed point $y^{*} \in S_{1}$ such that $y^{*}=\mathscr{F} y^{*}$ holds. Let $x^{*}(t)=t^{\alpha-1} y^{*}(t)$; then

$$
\begin{aligned}
x^{*}(t)= & t^{\alpha-1} P_{\alpha}(t) x_{0} \\
& +\int_{0}^{t}(t-s)^{\alpha-1} P_{\alpha}(t-s) f\left(s, x^{*}(s)\right) d s,
\end{aligned}
$$

$$
t>0,
$$

and, therefore, $x^{*}$ is a mild solution of (1). 

have

Noting that, for any $x \in \widetilde{S}_{1}$, using the condition (H1), we

$$
\begin{aligned}
\lim _{t \rightarrow \infty}|x(t)| \leq & \lim _{t \rightarrow \infty} \frac{M\left|x_{0}\right|}{\Gamma(\alpha)} t^{\alpha-1} \\
& +\lim _{t \rightarrow \infty} \frac{M K \Gamma\left(1-\beta_{1}\right)}{\Gamma\left(1+\alpha-\beta_{1}\right)} t^{\alpha-\beta_{1}}=0
\end{aligned}
$$

which yields that $x^{*}(t) \rightarrow 0$ as $t \rightarrow \infty$. Thus, the solution $x^{*}(t)$ is attractive.

Theorem 12. Assume that $Q(t)(t>0)$ is compact, and function $f \in C_{\alpha}(J, X)$ satisfies the following condition:

(H2) There exist $L \geq 0, \mu>0$ and $\beta_{2}>0$, such that $|f(t, x(t))| \leq L t^{\beta_{2}-1} e^{-\mu t}$, for any $x \in C((0, \infty), X)$.

Then the Cauchy problem (1) admits at least one attractive solution.

Proof. Let $\gamma_{2} \in(0,1)$. Then there exist constants $C=$ $C\left(\alpha, \beta_{2}, \mu\right)$ and $T_{2}>0$ such that

$$
\frac{M\left|x_{0}\right|}{\Gamma(\alpha)} T_{2}^{\gamma_{2}-1}+\frac{M L C}{\Gamma(\alpha)} T_{2}^{\gamma_{2}-1} \leq 1
$$

Let

$$
S_{2}=\left\{y: y \in E,\left|\frac{t^{\gamma_{2}} y(t)}{1+t}\right| \leq 1, \text { for } t \geq T_{2}\right\} .
$$

For any $y \in S_{2}$, let $x(t)=t^{\alpha-1} y(t), t \in(0, \infty)$. Then $x \in \widetilde{S}_{2}$, where

$$
\begin{aligned}
& \widetilde{S}_{2} \\
& =\left\{x \in C_{\alpha}(J, X):\left|\frac{t_{2} t^{1-\alpha} x(t)}{1+t}\right| \leq 1, \text { for } t \geq T_{2}\right\} .
\end{aligned}
$$

By (H2) and Lemma 8, similar to (39), we get

$$
\begin{aligned}
\left|\frac{t^{\gamma_{2}}(\mathscr{F} y)(t)}{1+t}\right| & \\
\leq & \frac{t^{\gamma_{2}}\left|P_{\alpha}(t) x_{0}\right|}{1+t} \\
& \quad+\int_{0}^{t} \frac{t^{1+\gamma_{2}-\alpha}(t-s)^{\alpha-1}}{1+t}\left|P_{\alpha}(t-s) f(s, x(s))\right| d s \\
\leq & \frac{M\left|x_{0}\right|}{\Gamma(\alpha)} \frac{t^{\gamma_{2}}}{1+t} \\
& \quad+\frac{M L}{\Gamma(\alpha)} \frac{t^{1+\gamma_{2}-\alpha}}{1+t} \int_{0}^{t}(t-s)^{\alpha-1} s^{\beta_{2}-1} e^{-\mu s} d s \\
\leq & \frac{M\left|x_{0}\right|}{\Gamma(\alpha)} t^{\gamma_{2}-1}+\frac{M L C}{\Gamma(\alpha)} t^{\gamma_{2}-1},
\end{aligned}
$$

for $t \geq T_{2}$, together inequity (52) and $0<\gamma_{2}<1$ yield that

$$
\begin{aligned}
& \frac{M\left|x_{0}\right|}{\Gamma(\alpha)} t^{\gamma_{2}-1}+\frac{M L C}{\Gamma(\alpha)} t^{\gamma_{2}-1} \\
& \quad \leq \frac{M\left|x_{0}\right|}{\Gamma(\alpha)} T_{2}^{\gamma_{2}-1}+\frac{M L C}{\Gamma(\alpha)} T_{2}^{\gamma_{2}-1} \leq 1 .
\end{aligned}
$$

Then, for $t \geq T_{2}$, from (55), we have

$$
\left|\frac{t^{\gamma_{2}}(\mathscr{F} y)(t)}{1+t}\right| \leq \frac{M\left|x_{0}\right|}{\Gamma(\alpha)} t^{\gamma_{2}-1}+\frac{M L C}{\Gamma(\alpha)} t^{\gamma_{2}-1} \leq 1,
$$

which deduce that $\mathscr{F} S_{2} \subset S_{2}$ for $t \geq T_{2}$.

The remaining part of the proof is similar to that of Theorem 11, and we omit it.

\section{Example}

Example 1. Let $X=L^{2}([0, \pi], \mathbb{R})$. Consider the following Cauchy problem for fractional partial differential equations:

$$
\begin{aligned}
&{ }^{L} D_{0+}^{\alpha} u(t, z)=\partial_{z}^{2} u(t, z)+\partial_{z} G(t, u(t, z)), \\
& z \in[0, \pi], t \in(0, \infty), \\
& u(t, 0)= u(t, \pi)=0, \quad t \in(0, \infty), \\
& I_{0+}^{1-\alpha} u(0, z)=u_{0}(z), \quad z \in[0, \pi],
\end{aligned}
$$

where $0<\alpha<1, G$ is a given function, $u_{0}(z) \in X$.

We define an operator $A$ by $A v=v^{\prime \prime}$ with the domain

$$
\begin{aligned}
& D(A)=\left\{v \in X: v, v^{\prime} \text { absoluty continuous, } v^{\prime \prime}\right. \\
& \quad \in X, v(0)=v(\pi)=0\} .
\end{aligned}
$$

Then $A$ generates a compact, analytic, self-adjoint $C_{0^{-}}$ semigroup $\{Q(t)\}_{t>0}$.

Let $x(t)=u(t, \cdot)$; that is, $x(t)(z)=u(t, z), t \in(0, \infty)$, $z \in[0, \pi]$. And the function $f:(0, \infty) \times X \rightarrow X$ is given by

$$
f(t, x(t))(z)=\partial_{z} G(t, u(t, z)) .
$$

Then the Cauchy problem (58) can be rewritten as the following format in $X$ :

$$
\begin{aligned}
{ }^{L} D_{0+}^{\alpha} x(t) & =A x(t)+f(t, x(t)), \quad t \in(0, \infty), \\
I_{0+}^{1-\alpha} x(0) & =x_{0},
\end{aligned}
$$

We can take $\alpha=1 / 4$ and $f(t, x(t))=t^{-1 / 3} \sin x(t)$. Then, (H1) is satisfied. According to Theorem 11, problem (58) has at least one attractive solution.

However, for the following integer order differential evolution equations,

$$
\begin{aligned}
\frac{\partial}{\partial t} u(t, z) & =\frac{\partial^{2}}{\partial z^{2}} u(t, z)+t^{-1 / 3}, \\
& z \in[0, \pi], t \in(0, \infty), \\
u(t, 0) & =u(t, \pi)=0, \quad t \in(0, \infty), \\
u(0, z) & =0, \quad z \in[0, \pi],
\end{aligned}
$$


As the argument described above, (62) can be rewritten as

$$
\begin{aligned}
& x^{\prime}(t)=A x(t)+t^{-1 / 3}, \quad t \in(0, \infty), \\
& x(0)=0
\end{aligned}
$$

Then (63) has an equivalent representation:

$$
x(t)=\frac{3}{2} t^{2 / 3}+\frac{3}{2} \int_{0}^{t} A \mathrm{e}^{A(t-s)} s^{2 / 3} d s,
$$

with $x(t) \rightarrow \infty$ as $t \rightarrow \infty$. This result shows that fractional evolution equations with Riemann-Liouville derivative have the global attractivity, whereas the integer order evolution equations do not have such attractivity.

\section{Conflicts of Interest}

The authors declare that they have no conflicts of interest.

\section{Acknowledgments}

The work was supported by the National Natural Science Foundation of China (no. 11671339).

\section{References}

[1] I. Podlubny, Fractional Differential Equations, Academic Press, San Diego, Calif, USA, 1999.

[2] A. A. Kilbas, H. M. Srivastava, and J. J. Trujillo, Theory and Applications of Fractional Differential Equations, Elsevier Science B.V., Amsterdam, Netherlands, 2006.

[3] K. Diethelm, The Analysis of Fractional Differential Equations, Lecture Notes in Mathematics, Springer, 2010.

[4] V. E. Tarasov, Fractional Dynamics: Application of Fractional Calculus to Dynamics of Particles, Fields and Media, Springer, 2010.

[5] Y.Zhou, Basic Theory of Fractional Differential Equations, World Scientific, Singapore, 2014.

[6] Y. Zhou, Fractional Evolution Equations and Inclusions: Analysis and Control, Elsevier, Academic Press, 2016.

[7] Y. Zhou, "Attractivity for fractional differential equations in Banach space," Applied Mathematics Letters, vol. 75, pp. 1-6, 2018.

[8] B. Ahmad, A. Alsaedi, M. Kirane, and R. G. Tapdigoglu, "An inverse problem for space and time fractional evolution equations with an involution perturbation," Quaestiones Mathematicae, vol. 40, no. 2, pp. 151-160, 2017.

[9] B. Ahmad, A. Alsaedi, and M. Kirane, "Nonexistence results for the Cauchy problem of time fractional nonlinear systems of thermo-elasticity," Mathematical Methods in the Applied Sciences, vol. 40, no. 12, pp. 4272-4279, 2017.

[10] Y. Zhou, L. Peng, B. Ahmad, and A. Alsaedi, “Topological properties of solution sets of fractional stochastic evolution inclusions," Advances in Difference Equations, vol. 2017, no. 1, article no. 90, 2017.

[11] Y. Zhou, B. Ahmad, and A. Alsaedi, "Existence of nonoscillatory solutions for fractional neutral differential equations," Applied Mathematics Letters, vol. 72, pp. 70-74, 2017.
[12] Y. Zhou and L. Peng, "Weak solutions of the time-fractional Navier-Stokes equations and optimal control," Computers \& Mathematics with Applications. An International Journal, vol. 73, no. 6, pp. 1016-1027, 2017.

[13] Y. Zhou and L. Peng, "On the time-fractional Navier-Stokes equations," Computers \& Mathematics with Applications. An International Journal, vol. 73, no. 6, pp. 874-891, 2017.

[14] Y. Zhou, V. Vijayakumar, and R. Murugesu, "Controllability for fractional evolution inclusions without compactness," Evolution Equations and Control Theory, vol. 4, no. 4, pp. 507-524, 2015.

[15] Y. Zhou and L. Zhang, "Existence and multiplicity results of homoclinic solutions for fractional Hamiltonian systems," Computers \& Mathematics with Applications. An International Journal, vol. 73, no. 6, pp. 1325-1345, 2017.

[16] I. Kim, K.-H. Kim, and S. Lim, "An $L_{q}\left(l_{p}\right)$-theory for the time fractional evolution equations with variable coefficients," Advances in Mathematics, vol. 306, pp. 123-176, 2017.

[17] J. Wang, M. Fečkan, and Y. Zhou, "A survey on impulsive fractional differential equations," Fractional Calculus and Applied Analysis, vol. 19, no. 4, pp. 806-831, 2016.

[18] J. Wang, M. Fečkan, and Y. Zhou, "Center stable manifold for planar fractional damped equations," Applied Mathematics and Computation, vol. 296, pp. 257-269, 2017.

[19] F. Chen, J. J. Nieto, and Y. Zhou, "Global attractivity for nonlinear fractional differential equations," Nonlinear Analysis: Real World Applications, vol. 13, no. 1, pp. 287-298, 2012.

[20] J. Losada, J. J. Nieto, and E. Pourhadi, "On the attractivity of solutions for a class of multi-term fractional functional differential equations," Journal of Computational and Applied Mathematics, vol. 312, pp. 2-12, 2017.

[21] J. Banaś and D. O'Regan, “On existence and local attractivity of solutions of a quadratic Volterra integral equation of fractional order," Journal of Mathematical Analysis and Applications, vol. 345, no. 1, pp. 573-582, 2008.

[22] E. Bazhlekova, "The abstract Cauchy problem for the fractional evolution equation," Fractional Calculus and Applied Analysis, vol. 1, no. 3, pp. 255-270, 1998.

[23] R.-N. Wang, D.-H. Chen, and T.-J. Xiao, "Abstract fractional Cauchy problems with almost sectorial operators," Journal of Differential Equations, vol. 252, no. 1, pp. 202-235, 2012.

[24] R. Zacher, "A De Giorgi-Nash type theorem for time fractional diffusion equations," Mathematische Annalen, vol. 356, no. 1, pp. 99-146, 2013.

[25] Y. Zhou, L. Zhang, and X. H. Shen, "Existence of mild solutions for fractional evolution equations," Journal of Integral Equations and Applications, vol. 25, no. 4, pp. 557-586, 2013.

[26] K. M. Furati, M. D. Kassim, and N. e. Tatar, "Existence and uniqueness for a problem involving Hilfer fractional derivative," Computers \& Mathematics with Applications. An International Journal, vol. 64, no. 6, pp. 1616-1626, 2012.

[27] C. Kou, H. Zhou, and Y. Yan, "Existence of solutions of initial value problems for nonlinear fractional differential equations on the half-axis," Nonlinear Analysis. Theory, Methods \& Applications. An International Multidisciplinary Journal, vol. 74, no. 17, pp. 5975-5986, 2011.

[28] R. P. Agarwal, Asma, V. Lupulescu, and D. O’Regan, “Fractional semilinear equations with causal operators," Revista de la Real Academia de Ciencias Exactas, Fisicas y Naturales - Serie A: Matematicas, vol. 111, no. 1, pp. 257-269, 2017. 
[29] B. M. Liu and L. S. Liu, "The decision theorems of relative compactness for two classes of abstract function groups in an infinte interval and its applications," Journal of Systems Science and Mathematical Sciences, vol. 30, no. 7, pp. 1008-1019, 2010. 


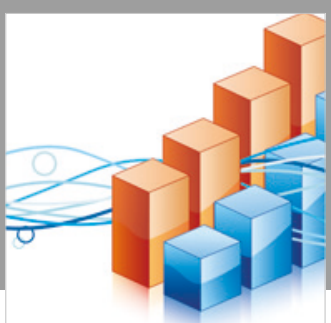

Advances in

Operations Research

\section{-n-m}
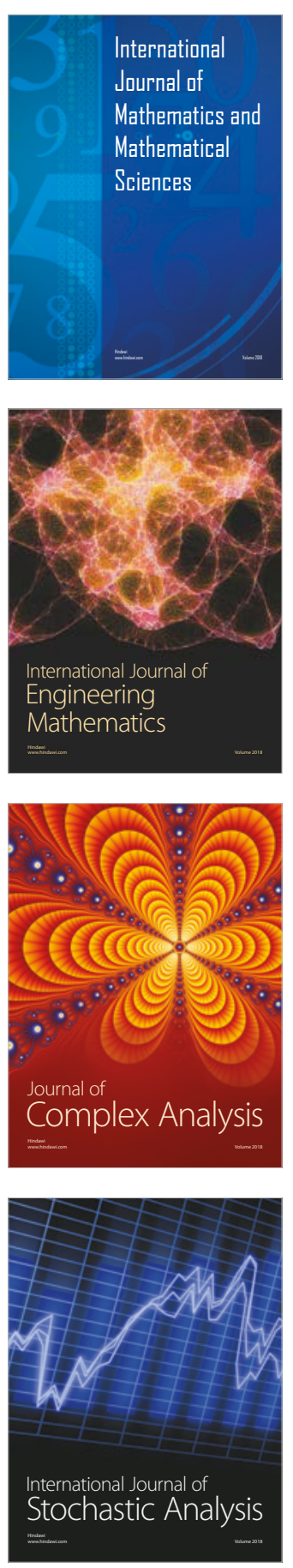
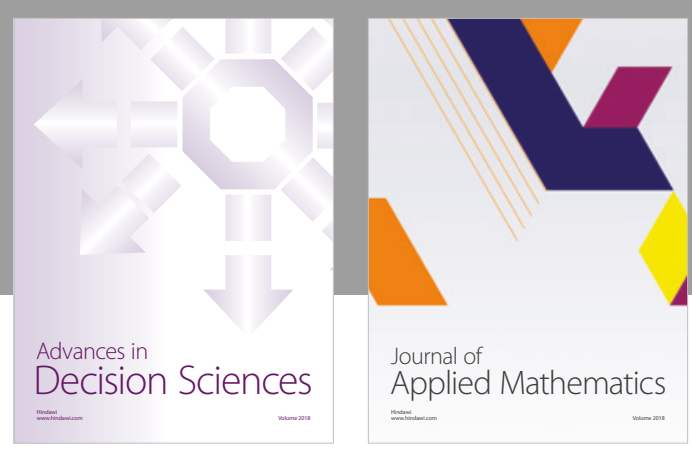

Journal of

Applied Mathematics
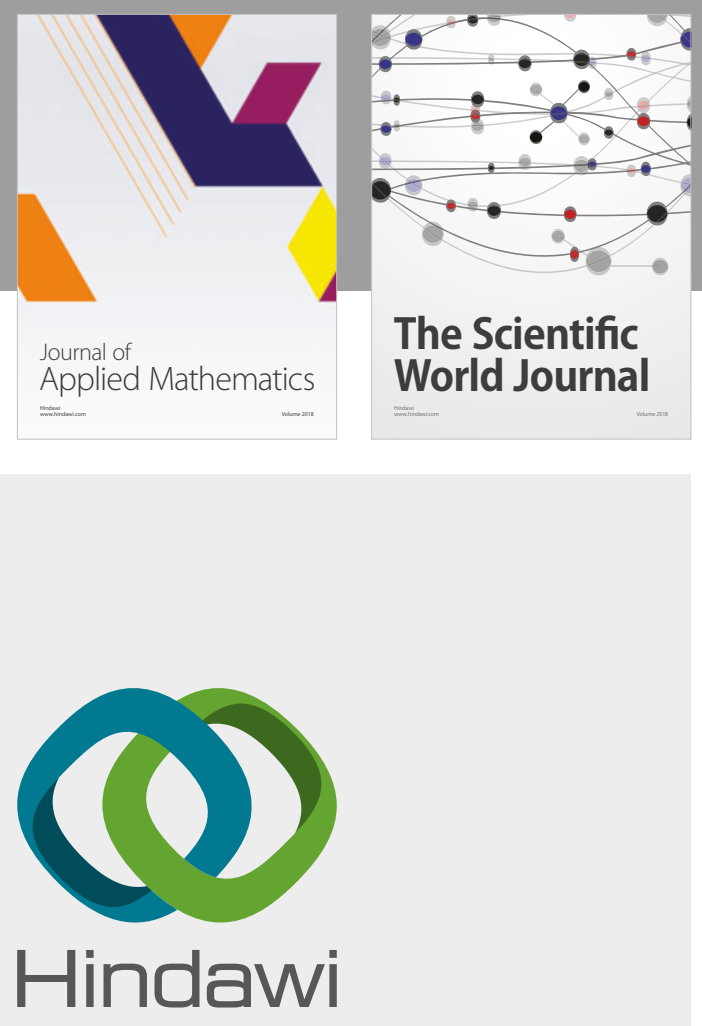

Submit your manuscripts at

www.hindawi.com

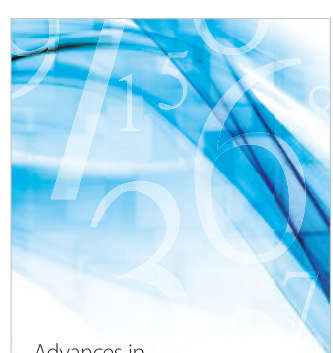

Advances in
Numerical Analysis
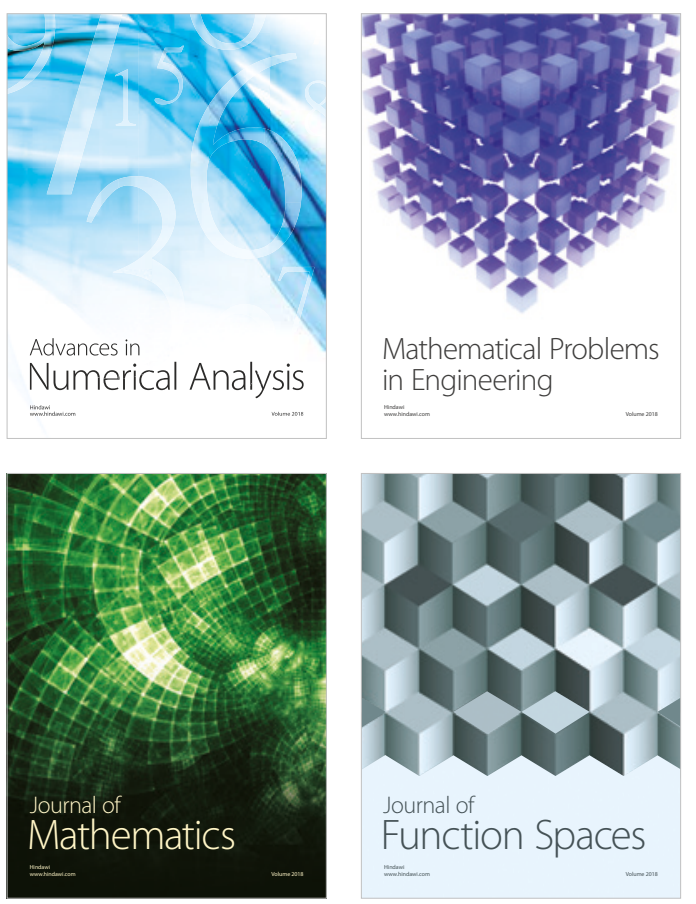

Mathematical Problems in Engineering

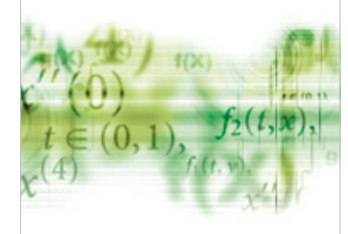

International Journal of

Differential Equations

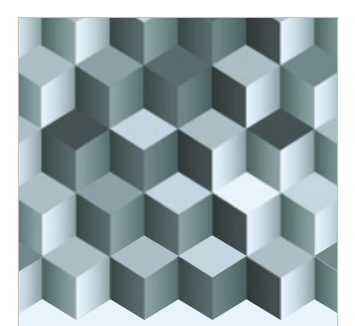

Journal of

Function Spaces

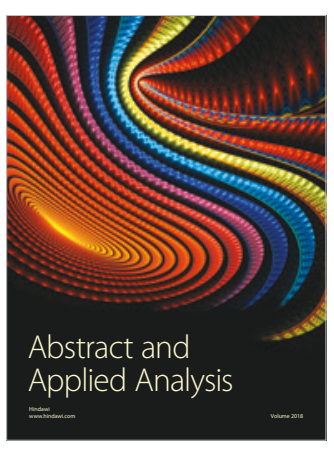

The Scientific

World Journal

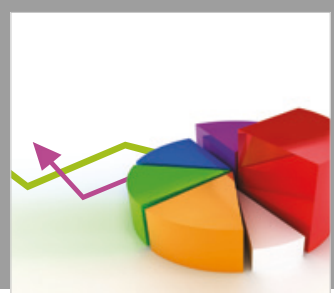

Journal of

Probability and Statistics
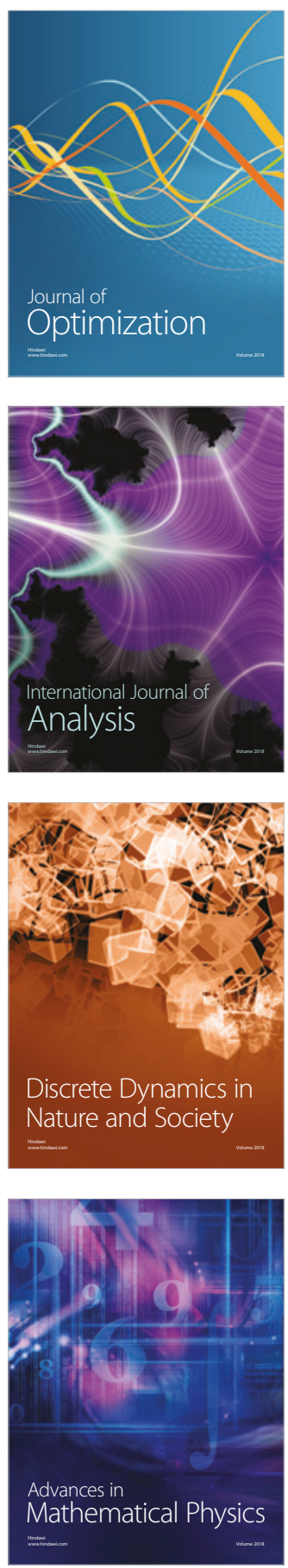